\title{
Practical Knowledge and Collaboration in Engineering
}

Päivi Pöyry ${ }^{1}$, Markus Mäkelä ${ }^{1}$, Jouni Meriluoto ${ }^{2}$ and Marju Luoma ${ }^{3}$

1) Helsinki University of Technology, Software Business and Engineering Institute, P.O.Box 9210, FIN-01025 HUT, Finland.

Email:ppoyry@soberit.hut.fi

2) Nokia Research Center, P.O.Box 407, FIN-00045, Nokia Group, Finland.

Email: Jouni.Meriluoto@nokia.com

3) Nokia Networks, P.O.Box 785, FIN-33101 Tampere, Finland.

Email:Marju.Luoma@nokia.com

Abstract: This paper presents the results of a case study conducted in networked product development organisations. In this study, the emphasis is on various dimensions of collaboration and knowledge sharing. The results indicate that knowledge is shared quite fluently in this network of collaborating partners. However, the tacit dimension of knowledge is not yet given enough attention. The information systems and other tools supporting knowledge management are focused on document management and other explicit knowledge, whilst the tactical knowledge is still shared in immediate communication or face-to-face meetings in an unstructured manner. The support for sharing tacit knowledge should be improved for example by encouraging the creation of communities of practice and by providing technological platforms for them.

Keywords: Knowledge management in engineering, Knowledge sharing, Knowledge creation, Collaboration, Distributed product development

\section{INTRODUCTION}

The conditions of product development engineering work have been changing during the last couple of years. Knowledge Management (KM) has become more important also in engineering. Keywords for today are global product development organisations, requirements for diverse, multiple and 
fast-changing competencies, strategic partnership networks, virtual teams, and improved organisational memory. The goal of $\mathrm{KM}$ in engineering is to facilitate the retain and reuse of information and knowledge created in the product development projects by passing it on to engineers within the project, to other projects and to collaborators. In this study, the information systems supporting knowledge management are expected to store and deliver product-specific information and to support re-using and sharing existing knowledge.

The purpose of this paper is to present the results of a case study conducted as a part of the WISE project. WISE (Web-enabled Information Services for Engineering) is a European Commission funded IST (Information Society Technologies) research project studying knowledge management within engineering environments.

In this study, the emphasis was on various dimensions of collaboration and knowledge sharing within and between organisations and people. We were especially interested in the facilitators and obstacles between strategic partnership companies. In addition, the work practices and work culture affecting the ways of sharing knowledge in the different organisations were investigated.

\section{KNOWLEDGE CREATION AND SHARING IN COLLABORATION}

The concept of managing knowledge has become increasingly popular both in the practical and in the academic discussion in the fields of engineering and management. Successful management of knowledge-related resources of companies has been recognised as a key basis for acquiring competitive advantage and other organisational success (HUBER 1991), and the acquisition and application of knowledge has even been argued to constitute the focal role of organisations in the society (DYER, NOBEOKA 2000). Virtual communities have significant possibilities of enhancing how this occurs (BIEBER et al. 2002). Today's environment of multi-organisation collaboration in technology alliances calls for improved knowledge sharing and processing capabilities, while information technology has been steadily increasing its importance as a tool of KM (STEIN, ZWASS 1995).

Knowledge is dynamic in nature, because it is created in social interactions among individuals and organisations. Being dependent on time and place (NONAKA, TOYAMA, KONNO 2001), knowledge is context-specific. Knowledge exists in organisations in different forms some of which are tangible in nature while others take a more subtle form. Tangible forms of knowledge have been termed as "articulated" or explicit (HUBER 1991; 
HEDLUND 1994), while intangible forms have been labelled "tacit". Explicit knowledge can be expressed in a formal and systematic way, and it can be shared in various forms, such as data, diagrams, and text. Its processing, storing, transmission is relatively easy. Tacit knowledge is harder to formalise because it is local, contextual and closely tied to place. Reconstructing tacit knowledge in a new context is difficult if not impossible. The borderline between tacit and explicit can be viewed as always shifting (HEDLUND, NONAKA 1993; POLANYI 1962).

Tacit knowledge can only be shared through shared experience. Apprenticeship, for instance, is a common way of adopting and sharing tacit knowledge. However, the concept of tacit knowledge does not cover only learnt practical skills, but also intuitions, assumptions and beliefs not expressed publicly (NONAKA, TAKEUCHI 1995). It is an advantage to distinguish explicit but also implicit knowledge from tacit knowledge.

The process of knowledge creation is a continuous, self-transcending one in which the boundaries of the old self are crossed and one enters into a new self by acquiring a new context. Knowledge is created in the interactions between individuals and their environments. Knowledge sharing refers to the beginning of the knowledge creation process, where tacit knowledge is shared among the individuals through socialisation. Typically this happens in a team with members from various backgrounds (NONAKA, TAKEUCHI 1995). Later in this paper, we have distinct focuses on the creation of knowledge, on its storing and retrieving, on re-using it and on its' sharing. Our analysis is distinct from the majority of prior literature due to our focus on distributed and extended environments.

\section{THE CASE STUDY}

In this study, we focused on networks formed by strategic partner companies and the engineers collaborating within these networks of global hightech product development. Fourteen product development engineers and other professionals such as technical writers were interviewed in their working environments. The interviews were carried out in Finland, in the cities of Helsinki, Espoo and Tampere in the premises of the focal company and its partners.

The idea was to apply the research methods traditionally used in ethnography in order to gather rich data and to form a thorough understanding on the subject area, but a full-scale ethnography was not used due to the time limitations and other constraints related to doing research in industrial settings. Thus, a "rapid ethnographic" approach was chosen (MILLEN 2000). The principal research method was semi-structured thematic interview, and 
these were recorded and transcribed and finally analysed with qualitative content analysis techniques. Originally the interviews were complemented by observations in the workplace, but they proved to have little value in terms of giving new information on the themes studied. Thus, these observations were left out from the data after an initial analysis. In this case study, we investigated these central KM-related issues:

- Creating knowledge

- Storing and retrieving information

- Re-using knowledge from other people and projects

- Sharing knowledge between people and organisations

The interview themes and questions (the interview framework) were prepared and the transcribed interviews were analysed in a group of four consisting of representatives of the focal company and researchers of Helsinki University of Technology. Two members of the analysis group carried out the interviews and participated in transcribing the tapes. The members of the group first read through and analysed the interviews individually. Then a group session was organised so that the individual interpretations could be discussed and a consensus could be reached on the results. There was only little divergence in the interpretations, and the common view was easily reached.

The themes of the interviews supported the analysis, and the interview framework was used as a backbone according to which the interview data was processed. The data was asked the questions defined in the interview framework themes, and the answers found in the data were grouped under the rubrics formed on the basis of the themes. The basic unit of analysis was a set of sentences - including the instance of one sentence - forming a meaningful expression of the subject matter.

\section{THE RESULTS OF THE STUDY}

\subsection{Creating Knowledge}

Creating knowledge was understood in various ways depending on the interviewee. For example, to create knowledge referred to inventing some new product or method, to getting a new idea, to producing specifications and other documents, and to coding innovative software. The knowledge creation was seen as an interactive process between individuals and a team; the ideas formed in someone's mind were further refined in one's team, and the results of teamwork and discussions could be further refined as individual work. In most interviews the knowledge creation was located at the focal 
company; the work between the partner companies and the focal company was divided so that the latter would develop, create and make innovations (product architectures, specifications in some cases innovative, not always) related to the product, and the partners would implement some well-defined part of the product. On the other hand, competence on the environment of the focal company, products, and tools accumulated.

"...I'd say that we create knowledge when we create a new product. Then we make something new in a concrete sense. But of course we may invent new ways of doing something. I think that's creating knowledge too."

"Creating knowledge... I think it happens in your head, but you need the rest of the team for it. It's partly inventing something on your own but you have to work it out with the colleagues."

"...we usually implement the knowledge the other company has created. It's a clear division of work. They make the innovation and we do what we are asked to support them. Then of course we may create new ways of making our work in a better way, but that's a different story."

Most interviewees interpreted knowledge as being tied up to physical products and other artefacts, even as "implementation" of external knowledge. Creation was not seen as an initially iterative generation in discourse as in collaborative engineering. Also the engineering aspect emphasised practical "doing" that was going hand in hand with knowledge creation. That is supporting the new division of tacit knowledge.

\subsection{Storing and Retrieving Information}

In most cases the information management was handled with a shared storage for project documents to which all partners had an access. In the projects studied, the information or document exchange was highly regulated between the focal company and the partner(s). As a practical detail, version management was found problematic, and there was no way to verify whether the document had been read or not. In addition, the information in the documents as well as the documents themselves were often out-dated. The various responsibilities for up-dating and managing documents caused "detective work" especially for project managers who were trying to find the information needed in the current project. In some cases, project members informed via e-mail when there was a new document or a version containing bigger changes and thus, worth re-reading. In most cases people seemed to be used to asking a certain document is updated or not (usually by e-mail). 
"Yes, we do have a shared place for the project files. But because it depends on the people that upload the documents on the server, it does not always work as it should. The documents and information may be like year old, or even worse. You never know what you'll find there. Or then the most recent document version will not be found there at all, it's just lost in the e-mail with the other trash mail."

The most common procedures and means for information exchange included e-mails, mailing lists, shared databases, and circulating regular project reports. Due to the fact that people did not save the documents in the shared system, the documents and information had to be searched for from several places and persons. It was difficult to get people use the information systems. For this reason the newest information was not always available, and the documents could often be out-dated. Clearly, there seemed to be place for improvements in version and change management. Crucial was that either the updated version of a document, or the meta-information about the new version would reach the reader. The user accessing the document management system "never knew what to find" but she had an assumption whether it was valid or not.

\subsection{Re-using Knowledge from Others}

Most projects reused information from other and previous projects in some form or other (e.g. processes and templates). Many projects were based on the previous projects (e.g. new releases of an existing product), and some projects needed information from other (parallel) projects. According to our results, information from other projects was difficult to find: the interviewees did not know where it was and who could access it. In the opinion of the interviewees information should be stored in one place or at least it should be accessed though one portal. Even more important would be information system and tool integration. An informant of ours claimed that information reuse from systems required personal contacts:

"...I'd say that I find the information when I look for it. But it requires a lot of detective work. Because you never know where the information is stored and who can access it. And you have to ask from several persons before you find the person that can tell where the information is and who can use it..."

Information was reused from previous projects if the information was available. In too many cases it was known that a specific document with specific information existed, but it was not known where the document could be found. The old documents could be used as models and they could be modified according to the current project's needs. For example, project 
plans, templates, and experiences were reused. More information could be reused if it was available. The re-use of information or knowledge still dealt mainly with explicit knowledge and information that could be expressed in writing. However, reusing tacit knowledge was difficult, because it had proved to be almost impossible to capture and to store this kind of highly personal and contextual knowledge. Some parts of tacit knowledge (as practical skills) could be captured with e.g. video devices. Of course this would be just one method to help to share and learn those skills, not capturing and storing the knowledge itself.

Thus, completely virtual work with no face-to-face meetings was not considered feasible, because the current information systems were incapable of supporting the sharing of tacit knowledge. Tacit knowledge is local, contextual and closely tied to place; reconstructing tacit knowledge in a new context is difficult. The sharing of tacit knowledge could be facilitated by supporting communities of practice with the help of information platforms. However, when implicit knowledge is distinguished from tacit knowledge, it is possible to derive explicit knowledge from implicit, in discourse and collaboration.

"We usually work so that we move from release to another. So we can use the test cases etc. again in the next release. We can use it as a model and make a new version."

"...I use the process documents and the slides from other projects. It simply saves so much time when I don't have to make it always from the scratch..."

Explicit knowledge from history as well as current documents from other people were helping in new challenges. When storing and sharing this kind of knowledge, e-mail and CSCW (Computer Supported Co-operative Work) tools were an advantage. Although implicit knowledge is subjective, and has no firm factual basis, it is cognitive and therefore able to be made explicit. That's the case also on organisational level. Information and communication technology helps to move the borderline from implicit towards new explicit knowledge.

\subsection{Sharing Knowledge between People and Organisations}

The role of information systems was considered central in sharing knowledge. Information systems were used for sharing, storing and exchanging documents that contained the information and knowledge created in the project. However, the systems did not function properly and people did not use them as they were expected to because there was lots of out- 
dated information and for instance version management was unsatisfactory. Here we can see a chicken and egg situation, typical of many new systems: people do not use or update information in the system because the information in the system is not up to date.

In addition, there were too many systems available, which is confusing; one or two systems/portals would be enough for the interviewees. They also acknowledged that the information systems were not used for sharing informal information and knowledge, such as lessons learned during the project execution. People felt that today there were too many tools, the tools did not function properly and they were not user-friendly, and they were being changed too often. The interviewees wanted a centralised tool or portal through which the information and knowledge could be easily accessed. No more detective work for finding relevant documents or people should be necessary.

"At the moment we would not be able to work without the computer systems. They are basic tools for us. But they could be improved... and people should start using them as they are supposed to..."

"...they are rather fragmented. ... and what we hate is that the systems change each year. Just when you have learned to use the system someone tells you it will change. ... There should be some integration and then we build interfaces through which you can move information from one system to another."

The biggest barrier to knowledge sharing seemed to be the fact that key people were, or at least told they were too busy to share information and knowledge and to answer all the questions asked. This, of course, depended on the project and the project phase. Another problem was that there were too few personal contacts between people working in different organisations. Sometimes company borders, foreign language, and physical distance etc. could be seen as obstacles, but not as severe as the lack of time and contacts. Additionally, the overload of e-mails was seen as problematic: people could not cope with the information overflow and processing all the e-mail messages burdened the interviewees.

The constant lack of time was the major barrier to knowledge sharing in addition to the lacking personal contacts. Differences between cultures could hinder efficient sharing of knowledge; it became increasingly difficult to share knowledge the more "distant" the cultures were. Changes in the economic picture and business situation affected people's willingness to share knowledge; when the market situation was challenging, people wanted to keep the knowledge by themselves, i.e. did not communicate that much in general. Moreover, when extensive organisational changes occurred, the per- 
sonal networks could fall apart, which complicated the sharing of knowledge.

"...it is not about the people being umwilling to help. It's about lack of time. They just don't have the time to answer the questions. The lack of time is the biggest problem."

"A foreign partner is a more complicated case. The cultural difference makes it... A good example is the way in which people from different cultures admit that something does not work properly. ... it is a challenging task to find the problems."

"I feel that the cultural differences are the biggest [barriers]. Language and culture are things that make it more difficult. ...But I don't think that the geographical distance affects; it's the same if you speak on the phone to the neighbour building or to the other side of the earth."

The factors facilitating knowledge sharing included personal contacts that were created in face-to-face meetings. This enhanced the common feeling of trust, or "knowing the colleagues". These contacts between people collaborating in a product development project facilitated the knowledge sharing significantly as they formed a network of resources that could be utilised in different phases of the project. Secondly, good project managers with their own reasonable ways to coordinate the collaborated work were recognised as a factor improving knowledge sharing. Naturally, the agreed procedures between companies with regard to knowledge and information sharing were seen as facilitators.

Moreover, the current organisational culture facilitated knowledge sharing by allowing people to ask questions and to do the work together. However, the distance seemed to affect the success of knowledge sharing: it was easier to share knowledge when people were located geographically close. In addition, it was of course easier to communicate with the native language.

"...nice to meet them face-to-face that you know that what kind of a person you are working with. ... it is easier to contact if you have a problem."

"If you know the people, not only from the mail discussions, it helps. Then, if the tools worked right, it would be easier without the extra problems."

"It is easier to communicate in Finnish with the Finnish colleagues. You just don't ask if you have to do it in English." 


\subsection{The Effect of the Organisational and Social Structures on Knowledge Sharing}

Knowledge was shared in face-to-face situations and through information systems. The role of the information systems was to store the created documents and to enable the reuse and exchange of these knowledge assets. According to the interviewees, knowledge sharing was encouraged by setting common goals and deadlines for project members, but no direct incentives - that would be destined only for knowledge sharing - were used. The interviewees were not aware of established ways of sharing knowledge, e.g. the lessons learnt, which were informal knowledge in most cases, seemed to be poorly documented.

There were no significant differences between the focal company and the partners in the ways and culture of working. Due to a long partnership there were common or compatible ways of working together. However, in some cases there were problems with foreign companies due to different cultures, languages, or time zones.

There was no real "knowledge-border" between the different companies as one could have expected, and information and knowledge seemed to flow quite fluently across the company borders. However, one clear bottleneck was identified: Project managers and contact persons controlled the flow of information and knowledge. More direct contacts and communication would be needed between people working in the collaborated projects, since the project managers and contact persons were continuously too busy to answer all the questions directed to them. The partner companies did not always "dare to disturb the busy project manager" and thus suffered from insufficient knowledge. Information sharing was considered difficult due to cultural or geographical distance; meeting people face-to-face in the beginning of the project helped to create an atmosphere of trust that facilitated communication and knowledge sharing via e-mail and phone.

"...it's hard to find out about the experiences of the others, because we don't meet these people and nobody writes down the lessons they have learned..."

"...we have worked together for so many years that we know each others' ways almost as well as we were working for the same company. We have kind of grown together."

"Sometimes it's not nice to stay at work late in the evening just to have a net meeting with the colleagues in the States. The time difference is some kind of an obstacle." 
"...I personally don't see a knowledge border. There is a gap but it's not that bad. We usually get all knowledge we askfor, if we ask for it."

\section{CONCLUSIONS AND DISCUSSION}

The results of our interviews can be summarised in short: information and knowledge sharing happens today quite well in the studied network of partners collaborating in product development projects. However, the tacit dimension of knowledge is not given enough attention. The information systems and other tools supporting knowledge management are focused on storing, sharing and searching documents and other explicit knowledge, and the not so easily articulated tacit knowledge is shared in immediate communication or face-to-face meetings in an ad-hoc, unstructured manner. The support for sharing tacit knowledge should be improved for example by encouraging the creation of communities of practice and by providing technological platforms for them.

The organisational differences are not very disturbing between partners that share a history of collaboration. Instead, the processes, ways of working and even organisational cultures are very close to each other. The challenges of collaboration reside in the multi-cultural co-operation: language and different meanings attached to work can be obstacles for efficient project work.

The factors facilitating knowledge sharing include direct personal contacts between the engineers working in partner companies, and face-to-face meetings in the beginning of projects that require extensive virtual communication between distant sites. Information systems also facilitate knowledge sharing, but they need to be improved and integrated in order to support easy access to information and knowledge.

The support for re-using existing information and knowledge is necessary. Most of the information produced in previous or parallel projects is needed in order to be able to plan and run the new project efficiently. This includes also a learning dimension: the lessons learned during the projects may be passed on, and the documents, templates and project plans may be used as models that can be modified according to specific project needs.

When talking about organisational learning it is important to recognise the shifting borderline between implicit and tacit knowledge - non-explicit knowledge is much more difficult to reconstruct when we are talking about tacit, and not only implicit knowledge. ICT can be used to refine implicit knowledge for sharing - "deeper" tacit knowledge is possible to make organisational only by making people to collaborate in practice.

Sharing the knowledge and information created in the product development process is vital for the today's engineering work. Being capable of 
reusing information and knowledge created in other projects enables the engineers to avoid extra work and to build on the existing knowledge. A mere document management system is insufficient as being unable to support the exchange of informal and often tacit knowledge based on lessons learned, experiences and new practices evolved during a project. Instead, an information system with an integrated approach to knowledge management and engineering work is needed. This system, however, can be a combination of many systems. Furthermore, when implementing such as system, a well-justified KM introduction plan is needed in order not to complicate the engineers' work with another new information system. System development should be based on existing communities. In addition to creating viable systems supporting collaborative work, more emphasis must be laid on formalising, training and creating guidelines and coherent practices on how to use KM systems and portals, because human adaptation requires time, change of mindset etc.

\section{REFERENCES}

\section{BIEBER, M. et al.:}

Towards knowledge-sharing and learning in virtual professional communities.

In: The Proceedings of the 35th Annual Hawaii International Conference on System

Sciences.

Los Alamitos, CA et al.: IEEE Computer Society, 2002.

DYER, J. H.; NOBEOKA, K.:

Creating and managing a high-performance knowledge-sharing network: The Toyota case.

In: Strategic Management Journal,

Chichester, 21(2000)3, pp. 345-367.

HEDLUND, G.:

A model of knowledge management and the $\mathrm{N}$-form corporation.

In: Strategic Management Journal,

Chichester, 15(1994)SUMME/2287, pp. 73-90.

HEDLUND, G.; NONAKA, I.:

Models of knowledge management in the west and Japan.

In: Implementing Strategic Processes, Change, Learning, and Cooperation.

Eds.: LORANGE, P. et al.

London: Basil Blackwell, 1993, p. 117-144.

HUBER, G. P.:

Organizational learning: The contributing processes and the literature.

In: Organization Science,

Providence, RI, 2(1991)1, pp. 88-115. 
MILLEN, D. R.:

Rapid Ethnography: Time Deepening Strategies for HCI Field Research.

In: Interactive systems: processes, practices, methods, and techniques.

Ed.: BOYARSKI, David.

New York, NY: Association for Computing Machinery, 2000, pp. 280-286.

(Proceedings of Designing Interactive Systems DIS'00.)

NONAKA, I.; TAKEUCHI, H.:

Knowledge Creating Company.

New York, NY et al.: Oxford University Press, 1995.

NONAKA, I.; TOYAMA, R.; KONNO, N.:

SECI, Ba and leadership: A unified model of dynamic knowledge creation.

In: Managing Industrial Knowledge: Creation, Transfer and Utilization.

Eds.: NONAKA, I.; TEECE, D.

London: Sage, 2001, pp. 13-43.

POLANYI, M:

Personal Knowledge: Toward a Post-critical Philosophy.

New York, NY: Harper, 1962.

STEIN, E. W.; ZWASS, V.:

Actualizing organizational memory with information systems.

In: Information Systems Research,

Providence, RI, 6(1995)2, p. 85-117. 